\title{
An EEG alpha-detection, feedback stimulation, and data analysis system
}

\author{
ROBERT L. BOUDROT, DAVID M. GOODMAN, and THOMAS B. MULHOLLAND \\ Psychophysiology Laboratory, Veterans Administration Hospital, Bedford, Massachusetts 01730
}

\begin{abstract}
A two-channel general-purpose EEG alpha-detection system is described, along with the associated computer hardware and software. A specific application for the study of the human orienting response using alpha-contingent visual stimulation is presented. Limitations are discussed.
\end{abstract}

The suppression of posterior alpha rhythms $(8-13 \mathrm{~Hz})$ following visual stimulation has been used as a bioelectric sign of "visual attention" from the first reports of Berger (1929) to the present. Although there is disagreement on the neurophysiological mechanism of alpha and of alpha suppression, alpha "blocking," or attenuation is a reliable response to visual stimulation.

Despite the reliability of occurrence of an alphablocking response, quantitative aspects of the response, for example, its duration, are extremely variable. This variability decreases the usefulness of alpha-blocking measures as on-line indices of orienting to a visual stimulus. Feedback from the EEG response back to the stimulus was first used to reduce unwanted, unsystematic variation of alpha suppression and to improve the economies of sampling (Mulholland \& Runnals, 1962). With feedback, each alpha could be associated with a stimulus, which decreased the time required to obtain an adequate serial sample of 30-odd responses.

Other applications of feedback EEG have been developed that concentrate on the training of changes of different characteristics of the EEG with the aid of feedback information (see Johnson, 1977; Travis, Kondo, \& Knott, 1975, for reviews). However, the ability of subjects to enhance alpha above baseline levels has been questioned, as has the existence of a subjective alpha experiential state (Plotkin, 1977). Nevertheless, although alpha feedback is of questionable utility in a training paradigm, it has been shown to be an efficient and useful methodological tool in the process of studying the orienting response (Mulholland, 1973). Reviews of the method of feedback electroencephalography for measuring the human orienting response (OR) have been presented (Boudrot, 1972; Goodman, 1973; Mulholland, 1968, 1973). This paper describes the current state of the method as used at the Bedford Veterans Administration Hospital Laboratory.

Several EEG biofeedback systems have been described (see Paskewitz, 1975, for a review) that are based on different signal-detection paradigms. Purely analog systems have been used by Boudrot (1972) and Pasquali (1969). Digital filtering in the time domain has been demonstrated by Beatty (1971), Paskewitz (1971), and Sharp, Smith, and Surwillo (1975) using digital computers. A combination of analog and digital techniques in period analysis was presented by Rouse (1975). Furthermore, clinically oriented biofeedback devices are now available commercially.

These systems differ in expense, ease of use, accuracy, reliability, and data recording capability. The alpha-detection system presented in this paper uses a relatively simple analog filtering amplitude threshold method that is inexpensive, compact, portable, and flexible.

The unique characteristic of the present system is found, not in the alpha-detection equipment itself, but, rather, in the methods and applications used.

\section{METHOD}

\section{System Overview}

The overall function of the system is to: (1) collect and store EEG alpha/no-alpha time durations from two scalp locations, (2) provide visual feedback contingent with the occurrence of alpha from one of the two hemispheric locations, and (3) manipulate, analyze, and graphically display the collected time durations. Figure 1 is a block diagram of the system.

\section{Apparatus}

Polygraph. The system uses a Grass Model 6 eight-channel EEG machine with inputs and outputs modified to be IRIG compatible. Two of the eight channels are used for event marking for record-keeping purposes. The 1VRMS outputs are used for driving the alpha detectors.

Alpha Detectors. Identical detectors are used for the simultaneous processing of two ongoing EEGs. Each detector consists of three components: (1) a bandpass filter (Krohn-Hite Model 3700 adjustable upper and lower cuttoff frequencies with an attenuation rate of $24 \mathrm{~dB} /$ octave outside the passband), (2) a Texas Instruments 741 operational amplifier with a variable-voltage gain from 0 to 10 for calibrating and normalizing purposes, and (3) an LFE Corporation 1-mA ac meter relay. The meter relay performs three functions: (1) meters signal for calibration, (2) performs a mean average deviation measurement on the signal, and (3) has a continuously variable set point that, when exceeded, closes the contacts of a normally open relay. This relay closure indicates that the criteria for alpha (frequency + amplitude) have been met. The response time for signal detection in each of the channels is approximately $.25 \mathrm{sec}$. A peak-reading voltmeter is used with each channel of EEG to 


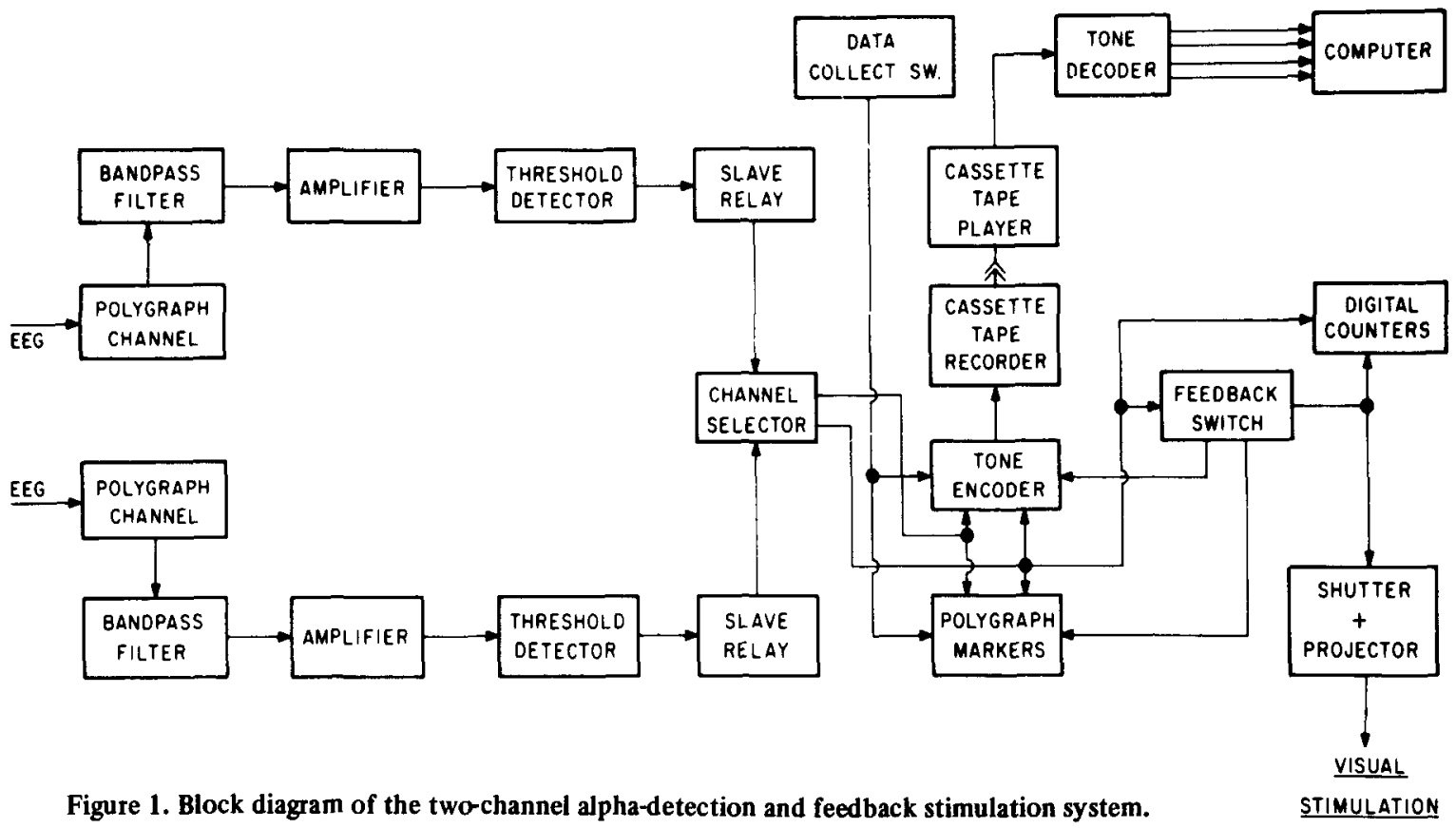

facilitate calibration and normalization. Digital counters (NonLinear Systems Model PC-4) keep track of the alpha/no-alpha events that occur in each channel. Switched low-voltage rectified ac is used to mark alpha durations and to indicate stimulus and epoch durations on the paper record.

Shutter/Projector. A Gerbrands electronic tachistoscopic shutter is used in conjunction with a Kodak Ektagraphic slide projector for the alpha-controlled presentation of visual stimuli.

Encoder/Decoder. When the system is used in a location remote from the computer, data are collected and stored on cassette tape and later read into the computer by way of a tone encoder/decoder system. Four solid state RC oscillators (Douglas Randall Fritch Driver), each with a distinct audio frequency, are keyed from (1) alpha-relay Channel 1, (2) alpha-relay Channel 2, (3) feedback stimulus occurrence, (4) data collectionenabled switch, and output to a standard cassette recorder/ player. The presence of the four recorded tones $(1,000,1,500$, $2,000,2,500 \mathrm{~Hz}$ ) indicates the state of the switch that each represents.

The frequency-encoded tape may then be decoded at the computer by a set of four corresponding frequency-sensitive switches (Douglass Randall Inc. Fritch).

\section{Computer Equipment}

A small laboratory computer (DEC PDP-12A) is used offline to time and record the durations of individual alpha and no-alpha events played back through the cassette tape tonedemodulator unit.

The computer, purchased in 1970, has been periodically updated, so that it now contains the following components: $16 \mathrm{~K}$ words of 12 -bit core memory, a 10 -megabyte moving head disk (System Industries Model 4500) with one fixed and one removable platter, a 30-character/sec teleprinter (DEC LA30 DECwriter), and a 100-dot/in. 500-line/min electrostatic printer/ plotter (Versatec Model $800 \mathrm{~A}$ ). Standard hardware features of the DEC PDP-12 computer that are utilized in these projects include an integral $7 \times 9$ in. refresh point-plot cathode-ray tube (CRT), 2 LINCtape drives (approximately $230 \mathrm{~K}$ words on each tape), a programmable crystal-controlled real-time clock (typically used at $100 \mathrm{~Hz}$ ), and 12 independent logic-level input (sense) lines that can also detect simple switch closures.

\section{Computer Software}

Two independent operating systems are used on the PDP-12 computer: DIAL-MS and OS/12, the latter being a more recent development that includes flexible device-independent operation, batch processing, more efficient hardware utilization, and an overlay-structured FORTRAN IV software system.

All data collection is performed under the DIAL system by the COLLECTR program and stored by file name on disk or tape by UTILITYS. Preliminary data reduction, graphical display, and least-squares curve fitting is performed by the DATAFIT program. More sophisticated data manipulation, statistical hypothesis testing, and automatic plotting of data are performed under the OS/12 operating system. The majority of these programs are written in FORTRAN IV. All computer software developed in this laboratory is available free of charge, although only the FORTRAN programs are directly transferable to other computer systems.

\section{Procedure}

EEG Recording. In a typical experiment, four monopolar EEG recordings are made (referenced to linked ear lobes) using chlorided Grass silver cup electrodes with a ground reference on the right mastoid process. One electrode is located at 10-20 position $0_{1}$, another at $0_{2}$, and the remaining two are located over Wernicke's area (left hemisphere plus contralateral homologue), as defined by (Matsumiya, Tagliasco, Lombroso, and Goodglass, 1972).

The EEG machine is calibrated with amplification and filtering as required, depending on the individual subject's EEG. The alpha-detection equipment is then calibrated using a procedure that serves to normalize the detection of alpha for each subject independently. The subject's alpha frequency is manually determined during an eyes-closed resting-in-the-dark baseline period; the high and low 3-dB cutoff frequencies on the bandpass filters are set at $1 \mathrm{~Hz}$ above and below that subject's alpha frequency, respectively. The variable-gain control in the alpha-detection equipment is then manipulated until the maximum peak-to-peak amplitude of the filtered EEG drives the meter relay to $80 \%$ of full scale during a visually observable alpha "burst" in the pen tracing. The amplitude threshold setting for a meter relay $O N$ is set at $25 \%$ of this maximum level. 
The peak-reading meters sample and hold the maximum amplitude reading and serve as convenient tools in performing the calibration. Thus, alpha is objectively defined as that signal which, after being filtered by the polygraph's and the alpha detector's bandpass filters, has at least $25 \%$ of the peak-to-peak amplitude of maximal baseline (eyes-closed) alpha.

This calibration procedure is performed on each EEG channel separately at the beginning of the experiment. It serves to reduce intersubject and interchannel EEG amplitude differences, normalizing all recordings such that the "facility" of alpha production for each subject and each recording site is equalized. If any EEG response changes are subsequently recorded in the experiment, they are more likely to be the result of the experimental manipulations, rather than the result of standing baseline EEG differences.

Following the calibration procedure, two baseline conditions are recorded, one with eyes closed and the other with eyes open in the dark. Each condition is initiated by turning on the data collection-enabled switch. The condition is manually terminated when at least 30 alpha and no-alpha "events" have been recorded in each of the two EEG channels. At least 10 alpha and no-alpha events are recorded with the subject's eyes open in the dark before the feedback switch is turned on. When the feedback switch is on, the visual stimulus is presented, contingent on the subject's alpha in one of the two EEG channels, the "contingent" channel. The stimulus is on for the duration of each alpha and off for each no-alpha event. The same stimulus is presented in a habituation paradigm on each occurrence of alpha in the contingent channel until at least $\mathbf{3 0}$ alpha and no- alpha events occur in both the contingent and noncontingent EEG channels. At this point, the feedback and data collection switches are turned off and the subject rests.

Eyes-closed and eyes-open baseline conditions are collected at the end of the experiment.

Data Collection. The general-purpose EEG alpha-feedback data collection program is COLLECTR (Goodman, 1973). A newer version of COLLECTR (COLLECTT), designed specifically for use with the off-line cassette tape system, operates using the following procedure.

The cassette tape containing the encoded audio tones is played back through the frequency switch decoding device, which continuously monitors the tape for the presence of each of the four audio tones. The on and off states of the two alpha relays and two control switches in the laboratory are duplicated in the same time relationship as they occurred when the experiment was in progress.

The tone decoder's four relays are interfaced to the computer through four sense-line inputs. COLLECTT samples the four sense lines every $20 \mathrm{msec}$ under control of the real-time clock. When the switch closure corresponding to the data collectionenabled switch is detected, COLLECTT records the durations of each alpha and no-alpha period in each of the two EEG channels under interrupt control (from clock "ticks"). COLLECTT simultaneously displays the collected data, using a background task on the computer's CRT in a form similar to that shown in Figure 2 (the time duration of the events is on the ordinate, and the ordinal positions of the alpha and noalpha events in the time series are on the abscissa). When the
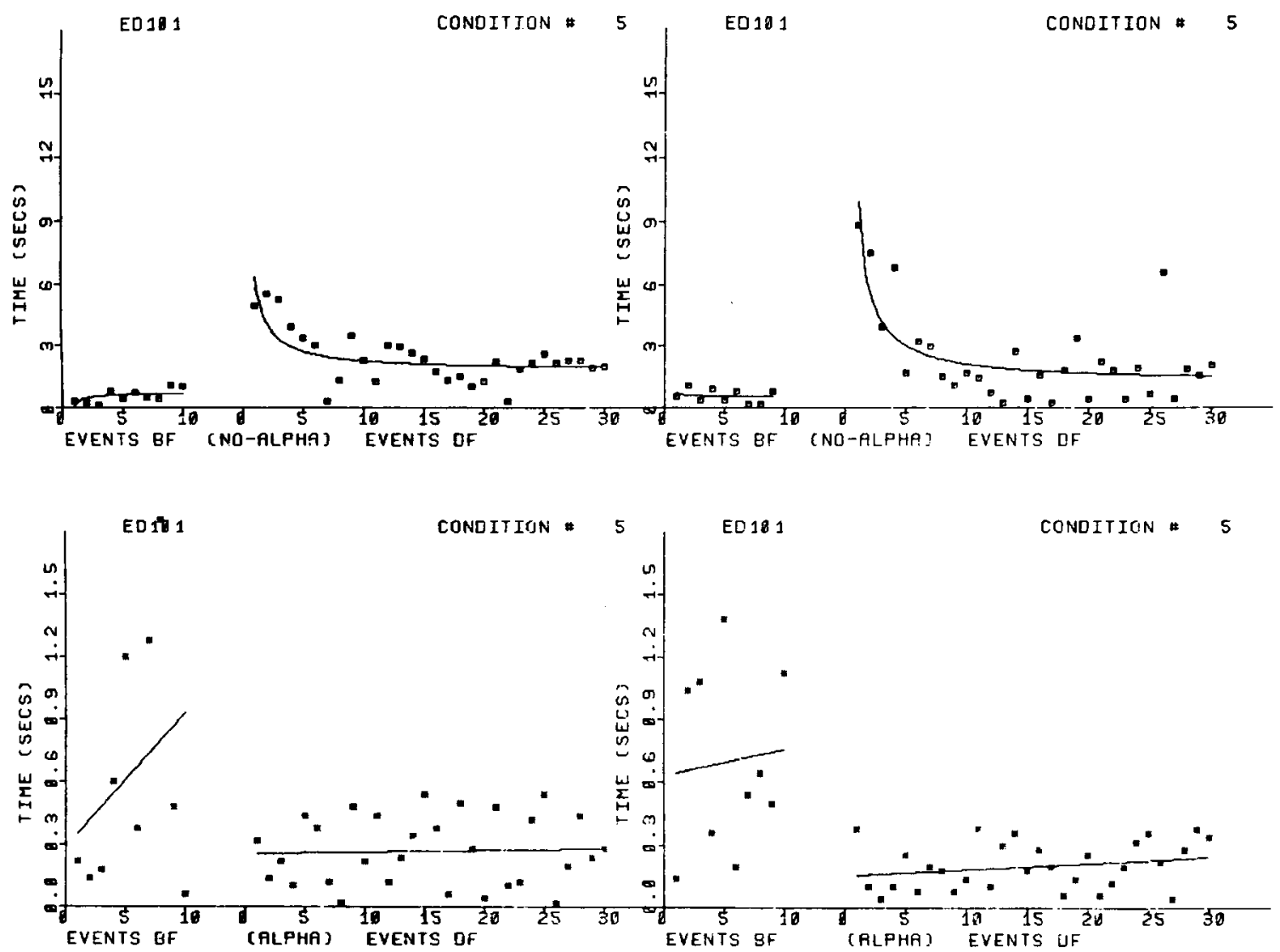

Figure 2. Scatterplot and best-fit habituation functions of alpha and no-alpha time durations before (EVENTS BF) and during (EVENTS DF) alpha-contingent visual feedback stimulation. Stimulation-controlling EEG channel $\left(\mathrm{O}_{2}-\mathrm{A}_{t}\right)$ is displayed in the left half of the figure, and noncontingent contralateral homologue data $\left(\mathrm{O}_{1}-\mathrm{A}_{t}\right)$ are displayed in the right half. Data are from an experimental diagnostics study, with the subject viewing the Hering "illusion." 
feedback switch closure is initially detected, COLLECTT identifies the first alpha burst in the stimulus-contingent EEG channel (i.e., the first alpha period associated with stimulation) and determines where within the current alpha or no-alpha period on the noncontingent EEG channel the start of stimulation occurs.

When the tone-decoder relay corresponding to the data collection-enabled switch opens, COLLECTT pauses $500 \mathrm{msec}$ and then checks that the switch is still off before terminating that condition and storing the data on the disk. The termination delay was found to be necessary to protect against intermittent erroneous loss of the audio tones on the cassette due to tape dropouts or other "glitches" and subsequent premature termination of a condition. The data from each condition are stored in separate 256-word blocks on the disk. Each block can hold up to 62 alpha/no-alpha pairs for two EEG channels, plus eight words of header information describing that condition, including the condition number and pointers to the first alpha events during feedback in each channel. If more than 62 event pairs are collected, COLLECTT automatically stores the full block of data on the disk and continues collecting into a new block without losing any data.

At the termination of the data collection, the experimenter uses the UTILITYS program to copy the new data into a permanent storage area on either LINCtape or disk under the DIAL operating system. All permanent data are file structured and accessed by unique names, thus preventing accidental erasure of data.

\section{Data Analy sis}

Graphical Display. After a patient's cassette tape containing the four audio tones has been carried back to the main laboratory and played back off-line into the computer using the COLLECTT program, the experimenter can set the PLOT operation into action. The PLOT programs combine FORTRAN and assembly language routines that are executed under an interactive batch-processing superstructure.

Figure 2 represents a graph generated by the PLOT procedure from data collected during an experimental diagnostics study. Some of the information that can be extracted from an inspection of the graphed data include the extent of initial orienting to the visual stimulus, the amount and rate of subsequent habituation, and the variability of the response.

The amplitude (duration) of the first few no-alpha events during the feedback-stimulated part of a condition indicates the extent of initial alpha blocking to the visual stimulus. A decrease in no-alpha durations until they reach asymptote represents normal habituation.

Descriptive Statistics. The amount of "raw" data collected during a complex experiment can quickly become unmanageable. For example, recording two EEG channels with 10 alpha and no-alpha events before feedback and 30 alpha/no-alpha pairs during feedback for each of 25 conditions (trials) for 30 subjects results in a total of 120,000 time duration data points. Fortunately, simple descriptive statistics can be calculated that adequately represent the important information contained in the data. Each condition in the experiment usually represents repetitive sampling of the EEG response to a single visual stimulus under one combination of the independent (treatment) variables being studied, using a randomized completely factorial design with repeated measures within a subject.

The primary descriptive statistics that are calculated separately for alpha and no-alpha events for each EEG channel for each condition (divided into before and during feedback subsections) are: the mean time duration (M), the standard deviation of the time durations (SD), and the ratio formed by dividing the calculated mean by the corresponding standard deviation. This control ratio (M/SD) is the reciprocal of the coefficient of variation and is a sensitive discriminator of the relative variability of the EEG response (Goodman, Note 1). Least-squares best-fit functions are calculated. For alpha, $\Delta \mathrm{ta}=(\mathrm{m})(\mathrm{N})+\mathrm{b}$, where $\Delta \mathrm{ta}$ is the time duration of a particular alpha burst in the habituation series, $N$ is the ordinal position of that alpha burst in the series, and $m$ and $b$ are the parameters of the best-fit function, respectively, the slope and the $y$-intercept. The best-fit function for no-alpha events is $\Delta \operatorname{tna}=(\mathrm{m})(1 / \mathrm{N})+\mathrm{b}$, where $\Delta$ tna is the time duration of a particular no-alpha period, $\mathrm{N}$ is the ordirial position of that no-alpha period in the habituation series, and $m$ and $b$ again are the slope and intercept of the best-fit function, respectively. In the no-alpha "linear" equation, the use of the reciprocal of $\mathrm{N}$ as the independent variable transforms the equation into that of a simple hyperbola in which $b$, the $y$-intercept, is more correctly thought of as an asymptote. The various parameters of the best-fit functions are the final descriptive statistics routinely calculated for each condition. These calculations are performed by the DATAFIT program, which also displays the raw data and best-fit function on the CRT.

Inferential Statistics. A package of FORTRAN analysis of variance (ANOVA) programs was written to facilitate the essential statistical process. These programs are flexible and general purpose in nature. The primary component of this software package is the ANOVA program, which accommodates up to 10 independent variables with any number of levels of each factor, with the restriction that the product of the number of levels of all factors be less than 600 . This number also corresponds to the maximum number of data points in the analysis. The factors can include any combination of nested and/or crossed relationships, including repeated measures on subjects within groups. The Millman and Glass (1967) algorithm is used to generate the sums of squares, but the determination of the expected mean squares, and thus the selection of the appropriate error term for use in the denominator of the $F$ ratios, is left up to the user, except in the instance of a factorial repeated-measures within-subjects (optionally, subjects-nested-within-groups) design. This design accommodates most of the experiments conducted in this laboratory. A separate program in the package generates for that design the names of all the sources of variance and their corresponding error terms in a form suitable for automatic input to the ANOVA program.

A disadvantage of this ANOVA program is that the optimization for core space is achieved at the loss of speed of operation. However, the processing of ANOVAs by this program can easily be performed in a batch configuration, and thus the lengthy computation-bound jobs can be accomplished in overnight batch runs without an operator in attendance.

A subsidiary program (MEAN) generates all means corresponding to a particular main effect or any interaction effects. A utility program (SHUFFL) is available to reorganize a set of data by removing certain factors or levels, or repositioning them so as to create "new" independent variables.

The above programs, together with a post hoc Duncan's Multiple-Comparisons Test (Kirk, 1968), comprise the statistical tests of significance usually found to be necessary.

\section{DISCUSSION}

The best-fit functions described previously are estimates of the trend of latency and duration of the alpha-suppression response during feedback stimulation (Mulholland, 1973; Mulholland \& Gascon, 1972).

The linear and hyperbolic functions are mathematically simple and provide an adequate and convenient description of the data. The validity of these functions as global indices of "attention" depends on the validity of EEG measures in general, with respect to their 
correlation with perceptual and cognitive functions. Clearly, EEG responses can index only salient, largescale cortical processes and are only indirect bioelectric signs of attentional processes.

Some OR researchers have used an amplitude-based measure of alpha blocking (Ali, 1972; Holloway \& Parsons, 1971), while the majority of experiments use alpha-block duration as the dependent variable (Novak, Diaz, \& Leinwand, 1973; Schaefer, 1975; Sharpless \& Jasper, 1956). Barry and Beh (1976) used both alpha amplitude and blocking duration as measures. Two problems with these studies are the use of subjective hand scoring of desynchronization duration and the choice of interstimulus interval. Sharpless and Jasper (1956) pointed out that the speed of habituation is largely dependent on the intertrial interval.

The system described in this paper resolves both of these methodological problems through the use of biofeedback as an experimental tool. An objective definition of alpha is established and maintained throughout an experiment. Durations of alpha and alpha blocking are preserved on cassette tape and read by computer. Stimuli are presented contingent on the subject's alpha with the intertrial interval equivalent to the alpha-blocking duration rather than at totally random intervals. The advantages of this feedback paradigm are twofold: Each stimulus is presented when the brain is in approximately the same state (alpha), and no stimuli are wasted. Each stimulus is directly associated with an alpha-blocking duration.

There is no evidence that feedback perse alters the functional relationship of alpha suppression to the stimulus, state, and subject variables that can modulate the attentional processes.

Recently, the control ratio of alpha and no-alpha durations (M/SD) has been used to test hypotheses about the replicative reliability of the transfer of the effects of the stimulus through afferent, integrative, and efferent CNS processes to the EEG. The rationale for this novel application is derived from a speculative analogy between feedhack experiments and feedback control systems (Mulholland, 1977a, 1977b, in press; Goodman, Note 1). These experiments have shown that a feedback closedloop experiment is more efficient for testing hypotheses about the replicative reliability of internal paths than a nonfeedback open-loop experiment.

\section{Limitations}

The feedback EEG method for studying the human OR has advantages compared to methods that do not use feedback: It is standardized, quantitative, economical, and on-line. On the other hand, it has limitations. These arise from the response being electroencephalographic, the relative specificity of the response to visual stimuli, and uncontrolled responses to visual aftereffects.

The method requires that alpha rhythms be detected. About $10 \%$ of a normal population exhibit little or no alpha rhythms and cannot be studied. The EEG may not be a valid index of $O R$ if the subject is taking tranquilizers, barbituates, or alcohol. Not only is the OR affected, but the spectral composition of the EEG in relation to the OR may be altered. Alpha is decreased if the subject is drowsy, so it is necessary to monitor the sleep-wakefulness continuum behaviorally and electroencephalographically, so that a drowsy no-alpha episode is not designated an alert no-alpha episode. The strict adherence to proper EEG technique must be followed, so that artifacts that may spuriously activate feedback can be avoided.

The establishment of alpha-amplitude thresholds for detection and for presentation of the stimulus is both critical and demanding. The normalization procedure in baseline recordings takes time and is sensitive to experimenter error.

Sources of stimulation that are not being tested must be controlled. The method is applicable, however, in moderately controlled environments, for example, an EEG clinic, although the unpredictable variability of the EEG response may increase.

Visual afterimages may cause uncontrolled variability of the durations of no-alpha periods (Barry \& Beh, 1976). This problem is not unique to feedback methods and can be reduced by a proper selection of target stimuli and of homogeneous background visual stimuli.

\section{REFERENCE NOTE}

1. Goodman, D. M. Detection of cerebral lateralization of function using EEG alpha feedback. Paper presented at the annual meeting of the Eastern Psychological Association, Washington, D.C., April 1, 1978.

\section{REFERENCES}

ALI, M. R. Pattern of EEG recovery under photic stimulation by light of different colors. Electroencephalography and Clinical Neurophysiology, 1972, 33, 332-335.

BARRY, R. J., \& BEH, H. C. EEG correlates of the afterimage of visual stimulation. Psychophysiology, 1976, 13, 75-80.

BEATTY, J. Effects of initial alpha wave abundance and operant training procedures on occipital alpha and beta wave activity. Psychonomic Science, 1971, 23, 197-199.

BERGER, H. Uber das Electrenkephalogram des Menschen. Archiv fur Psychiatrie and Nervenkrankheiten, 1929, 87, 527-570. [Translated in P. Gloor, Hans Berger on the electroencephalogram of man. Electroencephalography and Clinical Neurophysiology. 1969, Suppl. 28.]

Bounrot, $\mathbf{R}$. An alpha detection and feedback control system. Psychophysiology, 1972, 9, 461-466.

Goonman, D. M. ALFIE: Collection of EEG alpha under feedback control using time-series analysis. Psychophysiology, 1973, 10, 437-440.

Holloway, F. A., \& Parsons, O. A. Habituation of the orienting reflex in brain damaged patients. Psychophysiology, 1971, 8, 623-634.

Joknson, L. Learned control of brain wave activity. In J. Beatty \& H. Legewie (Eds.), Biofeedback and behavior. New York: Plenum, 1977. 
KIRK, R. E. Experimental design: Procedures for the behavioral sciences. Belmont, Calif: Brooks-Cole, 1968.

Matsumiya, Y., Tagliasco, V. Lombroso, C. T., \& Goodglass, H. Auditory evoked response: Meaningfulness of stimuli and interhemispheric asymmetry. Science, 1972, 175, $790-792$.

Millman, J., \& Glass, G. V. Rules of thumb for writing the ANOVA table. Journal of Educational Measurement, 1967, 4. 41-51.

Mulholland, T. B. Feedback electroencephalography. Activitas Nervosa Superior, 1968, 10, 410-438.

Mulholland, T. B. Objective EEG methods for studying covert shifts of visual attention. In F. McGuigan \& R. Schoonover (Eds.), The psychophysiology of thinking. New York: Academic Press, 1973.

Mulholland, T. B. Biofeedback method for locating the most controlled response of EEG alpha to visual stimulation. In J. Beatty \& H. Legewie (Eds.), Biofeedback and behavior. New York: Plenum, 1977. (a)

Mulholland, T. B. Biofeedback as scientific method. In G. Schwartz \& J. Beatty (Eds.), Biofeedback: Theory and research. New York: Academic Press, 1977. (b)

Mulholland, T. B. Experiments and control systems: An analogy. In N. Birbaumer \& H. D. Kimmel (Eds.), Biofeedback and self-regulation. Hillsdale, N.J: Lawrence Erlbaum, in press.

Mulnolland, T. B., \& Gascon, G. A quantitative EEG index of the orienting response in children. Electroencephalography and Clinical Neurophysiology, 1972, 33, 295-301.

Mul.holland, T. B., \& RunNals, S. A stimulus-brain feedback system for evaluation of alertness. Journal of Psychology, 1962 54, 69-83.

Novak. S., Diaz, J., \& Leinwand, S. Latency of alpha blocking as a function of photic stimulation during the cardiac cycle. Psychophysiology, 1973, 10, 21-26.

PASkEwitz, D. A. A hybrid circuit to indicate the presence of alpha activity. Psychophysiology, 1971, 8, 107-112.

Paskewtiz, D. A. Biofeedback instrumentation: Soldering closed the loop. American Psychologist, 1975, 30, 371-378.

Pasquali, E. A relay controlled by alpha rhythm. Psychophysiology. 1969, 6, 207-208.

Plotkin, W. B. On the social psychology of experiential states associated with EEG alpha biofeedback training. In J. Beatty \& H. Legewie (Eds.), Biofeedback and behavior. New York: Plenum, 1977.

Rouse, L. O. On-line period analysis of EEG by time-toamplitude conversion (TAC). Psychophysiology, 1975, 12, 476-479.

SChAEFfer, A. B. Newborn responses to nonsignal auditory stimuli: I. Electroencephalographic desynchronization. Psychophysiology, 1975, 12, 359-366.

Skarp, F. H., Smith, G. W., \& Surwillo, W. W. PARIH: Period analysis of the electroencephalogram with recording of interval histograms of EEG half-wave durations. Psychophysiology, 1975, 12, 471-475.

Sharpless, S. \& JASPER, H. Habituation of the arousal reaction. Brain, 1956, 79, 655-680.

Travis, T. A., Kondo, C. Y., \& Knott, J. R. Alpha enhancement research: A review. Biological Psychiatry, 1975, 10, 69-89. 